\title{
A Noncommutative Version of the Minimal Supersymmetric Standard Model
}

\author{
Masato Arai *, Sami Saxell $\oplus^{\oplus}$ and Anca Tureanu $\theta^{*}$ \\ High Energy Physics Division, Department of Physical Sciences, University of Helsinki \\ and Helsinki Institute of Physics, P.O.Box 64, FIN-00014, Finland
}

\begin{abstract}
A minimal supersymmetric standard model on noncommutative space-time (NC MSSM) is proposed. The model fulfils the requirements of noncommutative gauge invariance and absence of anomaly. The existence of supersymmetry with a scale of its breaking lower than the noncommutative scale is crucial in order to achieve a consistent gauge symmetry breaking.
\end{abstract}

*masato.arai@helsinki.fi

†sami.saxell@helsinki.fi

†anca.tureanu@helsinki.fi 


\section{Introduction}

Quantum field theories (QFT) on noncommutative (NC) space-time have been subject to intensive research during recent years, especially after it was shown [1] that they can be obtained as low-energy limits of open string theory in an antisymmetric constant background field. The NC space-time is defined by the commutation relation

$$
\left[\hat{x}^{m}, \hat{x}^{n}\right]=i \Theta^{m n}
$$

where $\hat{x}^{m}$ are space-time coordinate operators and $\Theta^{m n}$ is a constant antisymmetric matrix. One way to realize field theory on this space-time is to replace the usual product between any fields with the Moyal star-product

$$
(f g)(\hat{x}) \longmapsto(f * g)(x)=\left.e^{\frac{i}{2} \Theta^{m n} \partial_{x_{m}} \partial_{y_{n}}} f(x) g(y)\right|_{x=y} .
$$

This procedure gives rise to new exotic features such as violation of Lorentz-symmetry and ultraviolet/infrared (UV/IR) mixing (for reviews see [2, 3]).

Since NC QFT arises as a low energy effective limit from string and D-brane theory, it has the potential to provide an attractive and motivated framework for physics beyond the standard model (SM). However, it is known that it is difficult to consider a realistic phenomenological model building due to a number of constraints imposed by noncommutativity. The main restrictions are from the mathematical (group theoretical) structure of the NC gauge theories [4, 5]. For instance, the only allowed gauge groups in the $\mathrm{NC}$ spacetime are $U_{*}(n)$, the NC generalization of the unitary groups $U(n)$. In Ref. [6], the restrictions imposed by noncommutativity were taken advantage of and a $\mathrm{NC}$ version of the SM based on the gauge group $U_{*}(3) \times U_{*}(2) \times U_{*}(1)$ was constructed. The model can be considered as a minimal NC realization of the SM. Indeed, it leads to the usual SM observable particle content at low energies. The corresponding symmetry reduction is achieved by the introduction of a scalar field which was called Higgsac field in Ref. [6], a term which we shall use also in this paper. In this model, the generator of $U(1)_{Y}$ - the hypercharge group of symmetry - is constructed from a linear combination of the generators of the trace- $U(1)$ (in the following we will write simply $\operatorname{tr}-U(1)$ ) subgroups of the factors in the gauge group $U_{*}(3) \times U_{*}(2) \times U_{*}(1)$. This model solved the standing problem of electric charge quantization observed in [7], in which it was shown that the only allowed charges for $U_{*}(1)$ matter are 0 and \pm 1 , and, as a byproduct, it led to all the electric charges of the leptons and quarks as unique solution.

While the classical action of this model has many desirable properties, it was later found out that it suffers from some serious problems, namely violation of unitarity, [8], existence of chiral anomaly [9, 10] (see also [11]) and problems related the hypercharge $U(1)_{Y}$ sector [12, 13, 14, 15]. Solutions to unitarity and chiral anomaly were proposed in [16] but the problems with the $U(1)_{Y}$ gauge field remain unsolved. Taking the one-loop corrections to the polarization tensor of the tr- $U(1)$ gauge field into account, the UV/IR mixing effect causes an unacceptable infrared singularity. Furthermore the $\operatorname{tr}-U(1)$ gauge field may become tachyonic and one of the massless polarizations gets lost. The latter implies that we observe vacuum 
birefringence, i.e., a polarization dependent propagation speed. This leads one to conclude that the $U_{Y}(1)$ gauge field in the NC SM cannot be treated as a photon. These problems are disastrous for phenomenology.

In an attempt to cure the latter problem, the model was extended in Ref. [11] to the gauge group $U_{\star}(4) \times U_{\star}(3) \times U_{\star}(2)$. The crucial idea behind this extension is to construct the $U_{Y}(1)$ gauge field from a traceless combination of $U_{*}(n)$ generators and to make the tr$U(1)$ parts suffering from the problems mentioned above decouple at low energies, leaving only a $S U(n)$ symmetry in the low energy effective action. Indeed, to achieve this purpose, the model in Ref.[11] used the fact that the one-loop coupling constant for $\operatorname{tr}-U(1)$ becomes logarithmically smaller as scale decreases. This running behavior of $\operatorname{tr}-U(1)$ is caused by the $\mathrm{UV} / \mathrm{IR}$ mixing. However, the running of the $\operatorname{tr}-U(1)$ coupling was shown to be too slow [15] for complete decoupling, consequently the extra $U(1)$ gauge field would cause non-acceptable effects at low energies. In addition, the statement that the UV/IR mixing affects only the $\operatorname{tr}-U(1)$ part and not the "NC $S U(n)$ " part is valid only for the two-point functions ("gluon" propagator). Indeed, the general picture arrived at in [13] is that the UV/IR mixing effects are given by correlation functions of open Wilson lines, which implies that 3- and 4-point functions involving "NC $S U(n)$ gluons" exhibit the phenomenon. The gauge $U_{\star}(n)$ theory was shown to be renormalizable up to one-loop level [17, 18], consequently it is not clear how to use the renormalization group equation and discuss the properties of the $\beta$-function for a theory whose renormalizability has not been fully proven. The fact that the leading log approximation works similarly to the commutative case may be a good educated guess, but may also prove wrong, since the UV/IR mixing affects different diagrams differently and the dominant diagrams in the UV are not the same as the ones which are dominant in the IR (and which do not even appear in the commutative case). We believe that the issue of the UV/IR mixing has still to be studied, in close connection with the renormalizability. Though in this paper we give a special attention to the problems of the tr- $U(1)$ subgroup of $U_{*}(n)$, which were shown to appear already at one-loop level, we consider that a clear-cut conclusion regarding the UV/IR mixing has not yet been reached.

The situation is changed if the theory has supersymmetry (SUSY). Thanks to SUSY, dangerous quantum corrections in the polarization tensor cancel. As a result, the supersymmetric theory with $\operatorname{tr}-U(1)$ gauge group does not have the infrared singularity, tachyonic mass nor the polarization problem for $\operatorname{tr}-U(1)$ gauge field. The dangerous contribution appears again if SUSY is broken, which is the case in a realistic model at low energies. A theory with soft SUSY breaking terms has been studied and it has been shown that the infrared singularity actually does not appear [12] though the other problems are still left [14, 15, 19]. However, as we argue in this paper the existence of unbroken supersymmetry at higher scales may suppress these effects enough to make noncommutative $\operatorname{tr}-U(1)$ field a viable candidate for the photon. It is also well known that in the noncommutative case a supersymmetric version of the theory has a chance to have no UV/IR mixing or be renormalizable - an example is the supersymmetric NC Wess-Zumino model [20]. These considerations serve as an additional motivation for constructing a supersymmetrised version of the NC SM.

In this paper, we propose a $\mathrm{NC}$ version of the minimal supersymmetric standard model 
(MSSM). The NC MSSM we construct is based on the NC SM of Ref. [6]. We would like to note that the matter content of our model is not the same as the commutative MSSM's one. Requirements that the theory has SUSY, NC gauge invariance and cancellation of anomaly lead us to the introduction of two new extra Higgs fields and two leptonic superfields compared to the commutative MSSM matter content. In addition, in order to achieve the gauge symmetry reduction, we introduce a Higgsac superfield which is a supersymmetric extension of the Higgsac field proposed in [16]. In the NC setting, these fields are inevitably introduced in addition to the commutative MSSM matter content, and thus we call our model a NC version of MSSM. Although the NC space-time with the commutation relation (1.1) violates the Lorentz invariance, the field theory on such a space-time possesses the so-called twisted-Poincaré symmetry [21. The generators of the latter symmetry satisfy the same algebra as the usual generators of the Poincaré symmetry. Thus the representations are identical and the particles in NC field theory are still classified by their mass and spin. In the case of supersymmetry on NC spacetime, a twisted version of the super Poincaré symmetry exists [22], which also justifies the use of the usual particles and their supersymmetric partners. We also briefly discuss the problem of the hypercharge $U_{Y}(1)$ gauge field in our model. This gauge field is a linear combination of $\operatorname{tr}-U(1)$ gauge fields and therefore the problems mentioned above appear after SUSY is broken. We discuss a possible solution to this problem.

There exists also an alternative approach to building a NC version of the SM [23] in which the Seiberg-Witten map is used to relate the NC gauge theory to a commutative one. The mapping is based on the expansion of the star-products in the Lagrangian. This allows one to write the Lagrangian of a NC version of the SM as the Lagrangian of the commutative SM plus an infinite number of $\Theta$-dependent terms. However, this expansion may miss out some important NC effects caused by the UV/IR mixing. An approach based on the Seiberg-Witten map would lead to a model different from the one that we describe in this paper.

The paper is organized as follows. In section 2 we construct and discuss the minimal supersymmetric version of the SM on the NC space-time. In section 3 we discuss quantum properties of the hypercharge $U_{Y}(1)$ part and SUSY breaking. Section 4 is devoted to summary and discussion.

\section{$2 \quad \mathrm{NC}$ MSSM}

In this section, we construct the non-commutative version of MSSM. First of all, we briefly explain the restrictions on model building in non-commutative gauge theory, which come from the noncommutativity that constrains the possible gauge groups and representations [4, 5]. As mentioned in the introduction, in non-commutative field theory, the only allowed gauge group in $\mathrm{NC}$ space is the unitary group whose Lie algebra is closed under the Moyal bracket $[A, B]_{*}=A * B-B * A$. The NC unitary group, denoted by $U_{*}(n)$, is obtained by insertion of the star-product between the $U(n)$ matrix valued functions. Especially, it is not possible to have a direct NC generalization of $S U(n)$ gauge groups, because in NC space the star-product will destroy the closure condition. Other restriction is that the charges of the matter fields 
under $U_{*}(1)$ are quantized to just $0, \pm 1[7]$.

In addition to these restrictions, there is the no-go theorem [5] stating that the representations of the $u_{*}(n)$ algebra are restricted to $n \times n$ hermitian matrices. Hence the gauge fields are in $n \times n$ matrix form, while the matter fields can only be in fundamental, adjoint or singlet states. Furthermore, matter fields can only transform non-trivially under at most two simple subgroups of any gauge group consisting of a product of simple groups. In other words, the matter fields cannot carry more than two NC gauge group charges.

The above restrictions cause problems when attempting to construct NC MSSM. The first restriction tells us that one has to start with the gauge group $U_{*}(3) \times U_{*}(2) \times U_{*}(1)$ as a minimal extension of the commutative MSSM gauge group $S U(3) \times S U(2) \times U_{Y}(1)$. The increase in the gauge group implies that there are two new additional neutral weak bosons and their superpartners in the theory. These two new states of supermultiplet must be sufficiently massive in order to be consistent with present experimental data. In addition, spontaneous symmetry breaking must take place to have the correct MSSM commutative gauge group at low energies. The second restriction is problematic for construction of the NC MSSM based on the gauge group $U_{*}(3) \times U_{*}(2) \times U_{*}(1)$ since the quarks should have fractional hypercharges. We also have to pay attention to the last restriction when we make charge assignments. For instance, if the left-handed quark belongs to a fundamental representation of $U_{*}(3)$ gauge group, it should be charged under only one of the other groups, i.e., anti-fundamental representation of $U_{*}(2)$ and 0 charge for $U_{*}(1)$, or singlet for $U_{*}(2)$ and -1 for $U_{*}(1)$.

In order to break the gauge group $U_{*}(3) \times U_{*}(2) \times U_{*}(1)$ to the $\mathrm{SM}$ one, its subgroups $U_{3}(1) \times U_{2}(1) \times U_{1}(1)$ where $U_{n}(1)$ is $\operatorname{tr}-U(1)$ part of $U_{*}(n)$, must be broken down to hypercharge gauge group $U(1)_{Y}$. A breaking mechanism was proposed in the construction of the NC SM 6] by introducing the scalar field charged under trace- $U(1)$ group of $U_{*}(n)$. This scalar field was called Higgsac. When the Higgsac develops a vacuum expectation value, the $\operatorname{tr}-U(1)$ part of $U_{*}(n)$ gauge symmetry is broken. Eventually at non-commutative parameter $\Theta \rightarrow 0$ limit, the remaining symmetry is $S U(n)$. If the Higgsac $\phi$ is charged under the $\Theta$-independent $U_{n}(1) \times U_{m}(1)$-part of $U_{*}(n) \times U_{*}(m), U_{n}(1) \times U_{m}(1)$ gauge group is broken down to a diagonal group $U(1)$. In the NC SM, two Higgsac fields are necessary to obtain the SM gauge group. One Higgsac breaks $U_{3}(1) \times U_{2}(1)$ to a diagonal subgroup $U(1)^{\prime}$ and the other Higgsac then produces a breaking of $U(1)^{\prime} \times U_{1}(1)$ to $U(1)_{Y}$. Non-zero vacuum expectation values of the two Higgsacs give masses for gauge bosons corresponding to the broken $U(1)$ generators while massless $U(1)_{Y}$ hypercharge gauge boson is realized as a linear combination of $\operatorname{tr}-U_{n}(1)$. However, the breaking by this Higgsac field causes a problem on the unitarity violation [8]. The unitarity violation is related to the fact that the symmetry reduction by the Higgsac fields is not a spontaneous one, since it transforms only under the $\Theta$-independent $U(1)$-part\$s. It is not a representation of the gauge group and thus the symmetry reduction through Higgsac fields is not a spontaneous symmetry breaking mechanism.

In the following we shall explain how the above restrictions are overcome and give com-

$\S$ In effect, the Higgsac field transforming under the $\operatorname{tr}-U(1)$ part of $U_{*}(n)$ is not an allowed representation in the NC case, according to the no-go theorem (see [16]) for details. 
plete spontaneous gauge symmetry breaking mechanism in construction of the NC MSSM. Our method is based on Refs. [6] and [16], but we discuss the construction by introducing the superfield on the non-commutative superspace.

\subsection{Superfield formalism}

The superfield in the commutative theory is a function of the superspace coordinated

$$
z^{M}=\left(x^{m}, \theta^{\mu}, \bar{\theta}_{\dot{\mu}}\right), \quad \mu, \dot{\mu}=1,2,
$$

where $m=0,1,2,3$ is the space-time index, $\theta^{\mu}\left(\bar{\theta}_{\dot{\mu}}\right)$ is a Grassmann coordinate, and $\mu(\dot{\mu})$ is Weyl spinor index. This superspace is easily generalized to the NC setting [4]. In the NC setting, these coordinates satisfy the following algebra

$$
\begin{gathered}
{\left[\hat{x}^{m}, \hat{x}^{n}\right]=i \Theta^{m n},} \\
{\left[\hat{x}^{m}, \hat{\theta}^{\mu}\right]=0,} \\
\left\{\hat{\theta}^{\mu}, \hat{\theta}^{\nu}\right\}=\left\{\hat{\bar{\theta} \dot{\mu}}, \hat{\hat{\theta}^{\dot{\nu}}}\right\}=\left\{\hat{\theta}^{\mu}, \hat{\hat{\theta}^{\dot{\mu}}}\right\}=0,
\end{gathered}
$$

where $\hat{\theta}$ and $\hat{\bar{\theta}}$ are Grassmann coordinate operators. The superfield is defined just as in the commutative case, and noncommutativity is imposed simply by inserting star-products (1.2) into the action instead of usual product as

$$
f g(\hat{x}, \hat{\theta}, \hat{\bar{\theta}}) \longmapsto(f * g)(x, \theta, \bar{\theta})=\left.e^{\frac{i}{2} \Theta^{m n} \partial_{x_{m}} \partial_{y_{n}}} f(x, \theta, \bar{\theta}) g(y, \theta, \bar{\theta})\right|_{x=y}
$$

Also the formulation of gauge theories in the superspace is performed just as in the commutative case except for star-products between superfields, and the restrictions to gauge groups and representations is the same as discussed above.

\subsection{Superpotential}

First we explain matter content and superpotential. After that, we shall explain how gauge symmetry reduction occurs, correct fractional charges for the quarks are induced in our model and the anomalies are canceled by introducing new matter fields. As mentioned earlier, according to the no-go theorem, all fields in a NC gauge theory must transform in the fundamental, anti-fundamental, adjoint or bi-fundamental representation. We assign the fields to the representation shown in Table 1 and construct a superpotential of the NC MSSM. In the assignment we are guided by the following requirements:

- matter content (especially fermions) and charge assignment should produce the SM (MSSM) hypercharges at low energies,

\footnotetext{
『In this paper we follow the notation of Ref. [24].
} 


\begin{tabular}{cccc}
\hline Chiral Superfield & $U_{\star}(3)$ & $U_{\star}(2)$ & $U_{\star}(1)$ \\
\hline$L_{i}$ & 1 & 2 & 0 \\
$\bar{E}_{i}$ & 1 & 1 & -1 \\
$Q_{i}$ & 3 & $\overline{2}$ & 0 \\
$\bar{U}_{i}$ & $\overline{3}$ & 1 & +1 \\
$\bar{D}_{i}$ & $\overline{3}$ & 1 & 0 \\
\hline$L_{i}^{\prime}$ & 1 & 2 & -1 \\
$L_{i}^{\prime \prime}$ & 1 & 2 & 0 \\
\hline$H_{1}$ & 1 & $\overline{2}$ & +1 \\
$H_{2}$ & 1 & 2 & -1 \\
$H_{3}$ & 1 & 2 & 0 \\
$H_{4}$ & 1 & $\overline{2}$ & 0 \\
\hline
\end{tabular}

Table 1: Matter content of MSSM. The index $i$ denotes the family.

- the theory should be free of anomalies,

- the theory (with matter content and gauge groups) should be minimal.

To satisfy these requirements, one is let to the following superpotentiall:

$$
\begin{aligned}
\mathcal{W}= & \lambda_{e}^{i j} H_{1} * L_{i} * E_{j}+\lambda_{u}^{i j} Q_{i} * H_{2} * \bar{U}_{j}+\lambda_{d}^{i j} Q_{i} * H_{3} * \bar{D}_{j}+\mu_{12} H_{1} * H_{2}+\mu_{34} H_{3} * H_{4} \\
& +\left(\alpha_{1}^{i j k} Q_{i} * L_{j} * \bar{D}_{k}+\alpha_{2}^{i} L_{i} * H_{4}+\alpha_{3}^{i} L_{i}^{\prime} * H_{1}+\alpha_{4}^{i} L_{i}^{\prime \prime} * H_{4}+\lambda_{L^{\prime \prime}}^{i j} H_{1} * L_{i}^{\prime \prime} * E_{j}\right),
\end{aligned}
$$

with the charge assignments as in Table 1 . The first five superfields in Table 1 correspond to the usual quarks and leptons of the SM. In order to give Yukawa terms to all the SM fermions we introduce an additional Higgs superfield $H_{3}$ besides the superfields $H_{1}$ and $H_{2}$ appearing in the commutative MSSM. This is because the superfield $H_{1}$ giving a down-type mass after electroweak symmetry breaking cannot couple to down-type quark due to the charge assignment imposed by noncommutativity. We also introduce the two leptonic chiral superfields $L^{\prime}$ and $L^{\prime \prime}$, which are necessary to cancel the anomaly as will be discussed below. The above charge assignments are also required to achieve the correct fractional charges for fermions at low energies as will be explained. We also introduce a fourth Higgs to avoid the Witten anomaly (at least it is necessary in the $\Theta \rightarrow 0$ limit). It gives a new $\mu$-term $\mu_{34} H_{3} * H_{4}$.

Note that the first two terms inside the parentheses break lepton number symmetry. In order to remove the effect of these terms we introduce $R$-parity and $R$-parity conservation.

\footnotetext{
"There is also another possible choice for the charge assignments of the quarks, leptons and Higgs fields that leads to the Standard Model fermion content under symmetry breaking. However, this choice requires four additional leptonic doublets to be included in order to cancel anomalies, while the the charge assignments in Table 1 require only two additional doublets.
} 
Under $R$-parity, we define

$$
\begin{aligned}
& L, \bar{E}, Q, \bar{U}, \bar{D} \rightarrow-(L, \bar{E}, Q, \bar{U}, \bar{D}) \\
& L^{\prime}, L^{\prime \prime} \rightarrow-\left(L^{\prime}, L^{\prime \prime}\right) \\
& H_{1}, H_{2}, H_{3}, H_{4} \rightarrow H_{1}, H_{2}, H_{3}, H_{4} \\
& \theta \rightarrow-\theta
\end{aligned}
$$

Then the first four terms inside the parentheses in Eq. (2.4) are removed, leaving only one Yukawa term that includes the new leptonic field $L^{\prime \prime}$. However, as will be seen below, the two leptonic fields $L^{\prime}$ and $L^{\prime \prime}$ can obtain masses through the condensation of the Higgsac superfield and thus this term can be neglected at low energies. Consequently, all the terms inside the parentheses can be dropped out at low energies and the low energy superpotential will include only the superfields of the commutative MSSM and two additional Higgses $H_{3}$ and $H_{4}$. They give new Yukawa coupling terms (third and fifth terms in Eq. (2.4)). The matter content differs from the commutative MSSM by two additional Higgs fields and two leptonic fields.

\subsection{Symmetry reduction}

In this subsection we explain how the $\mathrm{NC}$ gauge symmetry $U_{3}(1) \times U_{2}(1) \times U_{1}(1)$ which is a subgroup $U_{*}(3) \times U_{*}(2) \times U_{*}(1)$ is broken down to the hypercharge $U(1)_{Y}$ gauge group. As explained earlier, if this breaking is performed by introducing the Higgsac field as in the NC SM [6], which is only charged under $\operatorname{tr}-U_{n}(1)$ subgroup of $U_{*}(n)$, the condensation of the Higgsac field does not mean spontaneous symmetry breaking of $U_{*}(n)$ and problems with unitarity consequently arise. Here we propose a mechanism of truly spontaneous symmetry breaking.

There are two aspects to be considered in a consistent spontaneous symmetry breaking of the $\operatorname{tr}-U(1)$ parts of $U_{*}(3) \times U_{*}(2) \times U_{*}(1)$. Let one first mention that one Higgsac field cannot achieve in one step the breaking of all three tr- $U(1)$ subgroups, simply because it does not have enough degrees of freedom to provide mass for two gauge fields [25]. As a result, two Higgsac fields are needed. If the first is charged under $U_{2}(1) \times U_{*}(1)$, which are broken to a residual $U(1)^{\prime}$, then the second has to be charged under $U(1)^{\prime} \times U_{3}(1)$. However, at scales above the first symmetry breaking, this second Higgsac field is actually charged under $U_{3}(1) \times U_{2}(1) \times U_{1}(1)$, because the generator of $U(1)^{\prime}$ is a linear combination of $U_{2}(1)$ and $U_{*}(1)$. Thus, the Higgsac fields cannot be constructed without circumventing the no-go theorem, first because they have to be charged under such subgroup, that they cannot be representations of the whole gauge group $U_{*}(3) \times U_{*}(2) \times U_{*}(1)$, and second because one of the Higgsac fields has to be charged under three subgroups.

The way of circumventing the no-go theorem is based on the noncommutative generalization of the gauge invariant operators using Wilson lines [26], leading to the possibility of constructing tensorial representations of the noncommutative gauge groups [27]. For the case of $U_{*}(3) \times$ $U_{*}(2) \times U_{*}(1)$, this approach was initiated in [16].

Circumventing the no-go theorem 
For simplicity, first we shall construct a gauge covariant Higgsac superfield which breaks tr$U(1)$ part of $U_{*}(n)$ gauge group. In order to construct it, let us first refer to the commutative case and introduce a chiral superfield which is $n$-index antisymmetric representation under $U(n)$,

$$
\phi^{\left[i_{1} i_{2} \ldots i_{n}\right]}(y, \theta)
$$

which transforms under $U(n)$ as

$$
\phi^{\left[i_{1} i_{2} \ldots i_{n}\right]}(y, \theta) \rightarrow\left(\phi^{\left[i_{1} i_{2} \ldots i_{n}\right]}\right)^{U}(y, \theta)=U_{i_{1}^{\prime}}^{i_{1}} U_{i_{2}^{\prime}}^{i_{2}} \cdots U_{i_{n}^{\prime}}^{i_{n}} \phi^{\left[i_{1}^{\prime} i_{2}^{\prime} \ldots i_{n}^{\prime}\right]}
$$

where $y^{m}=x^{m}+i \theta \sigma^{m} \bar{\theta}$, and its contraction with epsilon tensor:

$$
\phi(y, \theta)=\frac{1}{n !} \epsilon_{i_{1} i_{2} \ldots i_{n}} \phi^{\left[i_{1} i_{2} \ldots i_{n}\right]}(y, \theta)
$$

which transforms as

$$
\phi(y, \theta) \rightarrow(\phi)^{U}(y, \theta)=\frac{1}{n !} \epsilon_{i_{1} i_{2} \ldots i_{n}} U_{i_{1}^{\prime}}^{i_{1}} U_{i_{2}^{\prime}}^{i_{2}} \cdots U_{i_{n}^{\prime}}^{i_{n}} \phi^{\left[i_{1}^{\prime} i_{2}^{\prime} \ldots i_{n}^{\prime}\right]}=(\operatorname{det} U) \phi=e^{\operatorname{tr} U} \phi .
$$

i.e. the latter chiral superfield has charge $n$ under $\operatorname{tr}-U(1)$ and in the commutative case can cause the breaking of the $U(n)$ gauge group to $S U(n)$ upon condensation.

However, from the no-go theorem, the straightforward noncommutative generalization of the $n$-index antisymmetric object (2.6) is not an allowed representation of $U_{*}(n)$, because $n$ group elements should act from the left:

$$
\phi^{\left[i_{1} i_{2} \ldots i_{n}\right]} \rightarrow\left(\phi^{\left[i_{1} i_{2} \ldots i_{n}\right]}\right)^{U} \equiv U_{i_{1}^{\prime}}^{i_{1}} * U_{i_{2}^{\prime}}^{i_{2}} * \cdots * U_{i_{n}^{\prime}}^{i_{n}} * \phi^{\left[i_{1}^{\prime} i_{2}^{\prime} \ldots i_{n}^{\prime}\right]},
$$

where $U$ is a $U_{*}(n)$ gauge group element. One can easily see that it does not satisfy group multiplication law, i.e.

$$
\left(\left(\phi^{\left[i_{1} i_{2} \ldots i_{n}\right]}\right)^{U}\right)^{V}=\left(\phi^{\left[i_{1} i_{2} \ldots i_{n}\right]}\right)^{V * U} .
$$

Here $V$ is also a gauge group element. Therefore we cannot treat (2.8) itself as a representation of $U_{*}(n)$.

In order to overcome this restriction, the proposal of Ref. [27] is to modify the gauge transformation (2.10) in a non-trivial, gauge-field-dependent way, so that the group multiplication law holds. Furthermore one also has to modify (2.8) to be a $U_{*}(n)$ gauge group representation. Such a gauge transformation can be constructed if the gauge transformation involves the noncommutative version of supersymmetric half-infinite Wilson line, $W$. In the commutative case, the supersymmetric Wilson line is constructed in Ref. [28], and then it has been generalized to non-commutative setting in Ref. [29]. The explicit form for the case of $U_{*}(n)$ gauge group is given by

$$
\begin{aligned}
W & =P_{*} \exp _{*}\left(g \int_{0}^{1} d \sigma \frac{d z^{A}(\sigma)}{d \sigma} A_{A}\right) \\
& =\mathbf{1}_{N}+\sum_{n=1}^{\infty} \frac{g^{n}}{n !} \int_{0}^{1} d \sigma_{1} \int_{\sigma_{1}}^{1} d \sigma_{2} \cdots \int_{0}^{1} d \sigma_{n} \frac{\partial z^{A_{1}}\left(\sigma_{1}\right)}{\partial \sigma_{1}} A_{A_{1}} * \cdots * \frac{\partial z^{A_{n}}\left(\sigma_{n}\right)}{\partial \sigma_{n}} A_{A_{n}} .
\end{aligned}
$$


Here $A_{A}$ is the super gauge connection and $z^{A}=e_{M}^{A} z^{M}$ are the flat superspace coordinates ( $A$ runs over Lorentz indices $a$, spinor indices $\alpha$ and $\dot{\alpha}$ ), where $e_{M}^{A}$ is a supervielbein. The supervielbein is

$$
e_{M}^{A} \equiv\left(\begin{array}{ccc}
\delta_{m}^{a} & 0 & 0 \\
-i \sigma_{\mu \dot{\nu}}^{a} \bar{\theta}^{\dot{\nu}} & \delta_{\mu}^{\alpha} & 0 \\
-i \theta^{\rho} \sigma_{\rho \dot{\nu}}^{a} \dot{\epsilon^{\dot{\mu}}} & 0 & \delta_{\dot{\alpha}}^{\dot{\mu}}
\end{array}\right)
$$

and the super gauge connections are given in terms of the unconstrained superfields $U$ and $V$ by

$$
\begin{gathered}
A_{\alpha}=-e^{V} D_{\alpha} e^{-V}, \quad A_{\dot{\alpha}}=-e^{U} \bar{D}_{\dot{\alpha}} e^{-U}, \\
A_{a}=\frac{1}{4} i \bar{\sigma}_{a}^{\dot{\beta} \alpha}\left(-D_{\alpha} A_{\dot{\beta}}-\bar{D}_{\dot{\beta}} A_{\alpha}+\left\{A_{\alpha}, A_{\dot{\beta}}\right\}\right)
\end{gathered}
$$

where $D_{a}, D_{\alpha}$ and $\bar{D}_{\dot{\alpha}}$ are covariant derivatives defined by

$$
\begin{gathered}
e_{A}^{M} \frac{\partial}{\partial z^{M}} \equiv D_{A}=\left(\partial_{a}, D_{\alpha}, \bar{D}_{\dot{\alpha}}\right) \\
D_{\alpha}=\frac{\partial}{\partial \theta^{\alpha}}+i \sigma_{\alpha \dot{\alpha}}^{m} \bar{\theta}^{\dot{\alpha}} \frac{\partial}{\partial z^{m}}, \quad \bar{D}^{\dot{\alpha}}=\frac{\partial}{\partial \bar{\theta}_{\dot{\alpha}}}+i \theta^{\alpha} \sigma_{\alpha \dot{\beta}}^{m} \epsilon^{\dot{\beta} \dot{\alpha}} \frac{\partial}{\partial z^{m}} .
\end{gathered}
$$

Fixing the gauge partially by demanding $U=0$, the super gauge connection can be written purely in terms of the vector superfield $V$ :

$$
\begin{gathered}
A_{\alpha}=-e^{V} D_{\alpha} e^{-V}, \quad A_{\dot{\alpha}}=0, \\
A_{a}=-\frac{1}{4} i \bar{\sigma}_{a}^{\dot{\beta} \alpha} \bar{D}_{\dot{\beta}} A_{\alpha} .
\end{gathered}
$$

Partial gauge fixing leaves the gauge freedom for the vector superfield as

$$
e_{*}^{V} \mapsto e_{*}^{-i \Lambda^{\dagger}} * e_{*}^{V} * e_{*}^{i \Lambda}, \quad \bar{D}_{\dot{\alpha}} \Lambda=0,
$$

and thus it can be identified as the superfield whose vector field component is the usual $\mathrm{NC}$ gauge boson. The Wilson line operator transforms under gauge transformations as

$$
W \mapsto e_{*}^{i g \Lambda\left(z_{1}\right)} * W * e_{*}^{-i g \Lambda\left(z_{2}\right)},
$$

where $z_{1}$ and $z_{2}$ are the endpoints of the contour. As in nonsupersymmetric case, the actual shape of the Wilson line is not important. Then if we choose a half infinite line that starts from infinity $z_{1}=(\infty, \theta, \bar{\theta})$ and ends in $z_{2}=z$ with $\Lambda\left(z_{1}\right) \rightarrow 0$ this transformation reduces to

$$
W(z) \mapsto W(z) * e_{*}^{-i g \Lambda(z)},
$$

i.e. the half-infinite Wilson line is an anti-fundamental object under $U_{*}(n)$.

Now, by using the supersymmetric half-infinite Wilson line, we can modify (2.8) to be a $U_{*}(n)$ gauge invariant superfield $\Phi(z)$, while still carrying charge $n$ under its tr- $U(1)$ part[16]:

$$
\Phi(z)=\frac{1}{n !} \epsilon_{i_{1} i_{2} \ldots i_{n}} W_{j_{1}}^{i_{1}}(z) * W_{j_{2}}^{i_{2}}(z) * \ldots * W_{j_{n}}^{i_{n}}(z) * \phi^{\left[j_{1} j_{2} . . j_{n}\right]}(y)
$$


where the modified gauge transformation of $\phi^{\left[i_{1} i_{2} . i_{n}\right]}$ is given as in [27]:

$$
\begin{aligned}
\phi^{\left[i_{1} i_{2} \ldots i_{n}\right]} \rightarrow & \left(U * W^{-1}\right)_{i_{n}^{\prime}}^{i_{n}} *\left(U * W^{-1}\right)_{i_{n-1}^{\prime}}^{i_{n-1}} * \cdots *\left(U * W^{-1}\right)_{i_{2}^{\prime}}^{i_{2}} *\left(U * W^{-1}\right)_{i_{1}^{\prime \prime}}^{i_{1}} \\
& * W_{i_{1}^{\prime \prime}}^{i_{1}^{\prime}} * W_{i_{2}^{\prime \prime}}^{i_{2}^{\prime}} * W_{i_{3}^{\prime \prime}}^{i_{3}^{\prime}} * \cdots * W_{i_{n}^{\prime \prime}}^{i_{n}^{\prime}} * \phi^{\left[i_{1}^{\prime \prime} i_{2}^{\prime \prime} \ldots i_{n}^{\prime \prime}\right]} .
\end{aligned}
$$

The expression given in Eq. (2.20) is a gauge invariant Higgsac superfield, and the condensation of this superfield causes the spontaneous breaking of $\operatorname{tr}-U(1) \operatorname{subgroup}$ of $U_{*}(n)$.

Note that for single index representation, say, fundamental representation, the gauge transformation law reduces to the one of normal non-commutative gauge transformation since the Wilson line trivially cancels:

$$
\phi^{i_{1}} \rightarrow\left(U * W^{-1}\right)_{i_{1}^{\prime}}^{i_{1}} * W_{i_{1}^{\prime \prime}}^{i_{1}^{\prime}} * \phi^{i_{1}^{\prime \prime}}=U_{i_{1}^{\prime}}^{i_{1}} * \phi^{i_{1}^{\prime}}
$$

By similar considerations, we can introduce a field charged under an arbitrary number of gauge groups, e.g., a field which is in "fundamental representation" of $U_{*}(l), U_{*}(m)$ and $U_{*}(n)$, based on the auxiliary field $\phi^{n m l}$, with the gauge transformation

$$
\phi^{n m l} \rightarrow\left(L * W_{L}^{-1}\right)_{l^{\prime}}^{l} *\left(M * W_{M}^{-1}\right)_{m^{\prime}}^{m} *\left(N * W_{N}^{-1}\right)_{n^{\prime}}^{n} *\left(W_{N}\right)_{n^{\prime \prime}}^{n^{\prime}} *\left(W_{M}\right)_{m^{\prime \prime}}^{m^{\prime}} *\left(W_{L}\right)_{l^{\prime \prime}}^{l^{\prime}} * \phi^{n^{\prime \prime} m^{\prime \prime} l^{\prime \prime}}
$$

where $l, m$ and $n$ denote the indices for gauge groups $U_{*}(l), U_{*}(m)$ and $U_{*}(n)$, respectively, $L, M$ and $N$ are corresponding gauge group elements, and $W_{L}, W_{M}$ and $W_{N}$ are the corresponding half-infinite Wilson lines. Although the auxiliary field (2.23) has a cumbersome transformation law and is not in any definite representation of the gauge group $U_{*}(l) \times U_{*}(m) \times U_{*}(n)$, it turns out that an object constructed similarly to (2.20), i.e.

$$
\Phi^{[i j k]}=\left(W_{N}\right)_{n}^{i} *\left(W_{M}\right)_{m}^{j} *\left(W_{L}\right)_{l}^{k} * \phi^{[n m l]}
$$

is invariant under $U_{*}(l) \times U_{*}(m) \times U_{*}(n)$. The introduction of $n$-index field representations and of representations for arbitrary numbers of noncommutative gauge groups by the modification of the gauge transformation implies evading the no-go theorem stated in Ref. [5]. A more detailed discussion of this interesting issues is in progress [30].

\section{Symmetry reduction mechanism}

Let us turn to explain how gauge symmetry reduction occurs by the Higgsac superfield (2.20). In order that $\Phi$ defined in (2.20) develops a vacuum expectation value, we introduce the following superpotential:

$$
\mathcal{W}(\Phi)=m^{2} \Phi-\frac{\lambda}{3} \Phi * \Phi * \Phi
$$

Assuming that the perturbative vacuum for the gauge field is given by the pure gauge configuration, i.e. $\langle V\rangle=0$, we have

$$
\langle\Phi\rangle=\langle\phi\rangle=\frac{m}{\sqrt{\lambda}}
$$


In the following we shall see that the $\operatorname{tr}-U(1)$ gauge field of $U_{*}(n)$ gauge theory has a mass at the perturbative vacuum (2.25). First we expand the $\Phi$-field in the NC parameter $\Theta$ and the coupling constant $g$ :

$$
\begin{aligned}
\Phi(z)= & (\operatorname{det} W) \phi \\
= & \left(1+g \int_{0}^{1} d \sigma \frac{d z^{A}}{d \sigma} \operatorname{tr} A_{A}+\frac{g^{2}}{2} \int_{0}^{1} d \sigma^{1} \int_{0}^{1} d \sigma^{2} \frac{d z^{A}\left(\sigma^{1}\right)}{d \sigma^{1}} \operatorname{tr} A_{A}\left(\sigma^{1}\right) \frac{d z^{B}\left(\sigma^{2}\right)}{d \sigma^{2}} \operatorname{tr} A_{B}\left(\sigma^{2}\right)\right) \phi \\
& +\ldots
\end{aligned}
$$

where $\phi=\frac{1}{n !} \epsilon_{i_{1} i_{2} \ldots i_{n}} \phi^{\left[i_{1} i_{2} \ldots i_{n}\right]}$. Here the superfields $A_{A}$ and $\phi$ are $\Theta$-independent and the ellipsis denotes terms that are at least of first order in $\Theta_{m n}$ or third order in $g$. Using the WessZumino gauge and equations (2.13) and (2.16), and choosing the contour so that the Grassmann coordinates are constant with respect to $\sigma$, we can simplify the integrand to

$$
\frac{d z^{A}}{d \sigma} \operatorname{tr} A_{A}=\frac{d z^{M}}{d \sigma} e_{M}^{A} \operatorname{tr} A_{A}=-n \frac{d x^{m}}{d \sigma} \frac{i}{4} \bar{\sigma}_{m}^{\dot{\beta} \alpha} \bar{D}_{\dot{\beta}} D_{\alpha} V^{0}
$$

where $V^{0}$ is the $\operatorname{tr}-U(1)$ part of the gauge vector superfield. Inserting this to the expansion (2.26) and using the commutation relation

$$
\left\{D_{\alpha}, \bar{D}_{\dot{\beta}}\right\}=-2 i \sigma_{\alpha \dot{\beta}}^{m} \partial_{m}
$$

one can obtain the following form for the kinetic term of the Higgsac superfield

$$
\int d^{2} \theta d^{2} \bar{\theta} \Phi^{\dagger}(z) \Phi(z)=\int d^{2} \theta d^{2} \bar{\theta} \phi^{\dagger}\left(1+n g V^{0}+\frac{1}{2}\left(n g V^{0}\right)^{2}\right) \phi+\mathcal{O}\left(\Theta^{m n}\right)+\mathcal{O}\left(g^{3}\right) .
$$

The first three terms in the right hand side represent the usual kinetic term for $\phi$ and its gauge interactions. The other terms provide the gauge invariant completion. It is now clear that if the field $\phi$ has a nonzero vacuum expectation value the $\operatorname{tr}-U(1)$ gauge boson and its superpartner gaugino have masses. Decomposing the Lagrangian (2.29), bosonic parts are

$$
\begin{gathered}
\int d^{2} \theta d^{2} \bar{\theta} \Phi^{\dagger}(z) \Phi(z)=F \bar{F}+\phi \square \bar{\phi}+i \partial_{m} \bar{\psi} \bar{\sigma}^{m} \psi+n g\left(\frac{1}{2} \bar{\psi} \bar{\sigma}^{m} \psi+\frac{i}{2} \bar{\phi} \partial_{m} \phi-\frac{i}{2} \partial_{m} \bar{\phi} \phi\right) \\
-\frac{i n g}{\sqrt{2}}(\phi \bar{\lambda} \bar{\psi}-\bar{\phi} \lambda \psi)+\frac{1}{2}\left(n g D-\frac{1}{2}(n g)^{2} A_{m} A^{m}\right)+\mathcal{O}\left(\Theta^{m n}\right)+\mathcal{O}\left(g^{3}\right) .
\end{gathered}
$$

Substituting the vacuum expectation value of $\phi(2.25)$ into the above equation, we obtain the following mass terms

$$
\mathcal{L}_{\text {mass }}=\frac{i m n g}{\sqrt{2 \lambda}}(\lambda \psi-\bar{\lambda} \bar{\psi})-\frac{g^{2} n^{2} m^{2}}{4 \lambda} A_{0}^{m} A_{0 m},
$$

where $\lambda, \psi$ and $A_{0}^{m}$ are a gaugino, a fermion in the superfield $\phi$ and a gauge boson, respectively. We emphasize that the modified version of the Higgsac mechanism leads to spontaneous symmetry breaking and thus it should not cause any problems with unitarity. However, proving 
this statement explicitly is highly nontrivial since all the terms in the expansion of $\Phi$ need to be considered.

Above we described the mechanism in the case of a single gauge group $U_{*}(n)$. In the $\mathrm{NC}$ MSSM we have to apply this mechanism to a direct product of $U_{*}(n)$ factors, $U_{*}(3) \times U_{*}(2) \times$ $U_{*}(1)$. In order to obtain SM gauge group, we need to break the direct product of gauge groups $U_{3}(1) \times U_{2}(1) \times U_{1}(1)$ which is subgroup of $U_{*}(3) \times U_{*}(2) \times U_{*}(1)$ to the hypercharge $U(1)_{Y}$ gauge group. Its breaking can be achieved by two Higgsac superfields as in the NC SM case. In principle, the first Higgsac superfield could be charged under any of the three factors, but for the economy of the model we shall take it to break $U_{2}(1) \times U_{*}(1)$ down to $U(1)^{\prime}$, and then the second Higgsac will break $U(1)^{\prime} \times U_{3}(1)$ down to $U(1)_{Y}$. This choice is motivated by the fact that we have introduced new matter fields, $L^{\prime}$ and $L^{\prime \prime}$, in order to obtain a vector-like spectrum under $U_{*}(2)$ and $U_{*}(1)$, as required by the anomaly cancelation condition. The Higgsac field charged under $U_{2}(1) \times U_{*}(1)$ will have also the role to give masses to the newly introduced matter fields.

Thus, the first composite Higgsac superfield will be carrying charge 2 coupled to tr- $U(1)$ of $U_{*}(2)$ and charge -1 coupled to $U_{*}(1)$ :

$$
\Phi(z)_{U_{*}(2) \times U_{*}(1)}=\frac{1}{2 !} \epsilon_{i_{1} i_{2}}\left(W_{U_{*}(2)}\right)_{j_{1}}^{i_{1}} *\left(W_{U_{*}(2)}\right)_{j_{2}}^{i_{2}} * \phi(z)_{k}^{\left[j_{1} j_{2}\right]},
$$

where $\phi(z)_{k}^{\left[j_{1} j_{2}\right]}$ transforms as

$$
\phi_{k}^{\left[j_{1} j_{2}\right]} \rightarrow\left(U_{2} * W_{U_{*}(2)}^{-1}\right)_{j_{2}^{\prime}}^{j_{2}} *\left(U_{2} * W_{U_{*}(2)}^{-1}\right)_{j_{1}^{\prime}}^{j_{1}} *\left(W_{U_{*}(2)}\right)_{j_{1}^{\prime \prime}}^{j_{1}^{\prime}} *\left(W_{U_{*}(2)}\right)_{j_{2}^{\prime \prime}}^{j_{2}^{\prime}} * \phi_{k}^{j_{1}^{\prime \prime} j_{2}^{\prime \prime}} *\left(U_{1}^{-1}\right)^{k}
$$

where $U_{2}$ is the element of $U_{*}(2)$ and $U_{1}$ is the element of $U_{*}(1)$.

The second Higgsac field, charged under tr- $U(1)$ of $U_{*}(2)$ and $U_{*}(3)$ and also under $U_{*}(1)$ (and after the first symmetry reduction, under $U(1)^{\prime} \times U_{3}(1)$ ) is given by

$$
\begin{aligned}
\Phi(z)_{U_{*}(3) \times U_{*}(2) \times U_{*}(1)} & =\frac{1}{2 ! 3 !} \epsilon_{i_{1} i_{2}} \epsilon^{l_{1} l_{2} l_{3}}\left(W_{U_{*}(2)}\right)_{j_{1}}^{i_{1}} *\left(W_{U_{*}(2)}\right)_{j_{2}}^{i_{2}} *\left(W_{U_{*}(1)}\right)_{k} \\
& * \phi(z)_{\left[n_{1} n_{2} n_{3}\right]}^{\left[j_{1} j_{2} k\right]} *\left(W_{U_{*}(3)}^{-1}\right)_{l_{1}}^{n_{1}} *\left(W_{U_{*}(3)}^{-1}\right)_{l_{2}}^{n_{2}} *\left(W_{U_{*}(3)}^{-1}\right)_{l_{3}}^{n_{3}},
\end{aligned}
$$

where

$$
\begin{aligned}
\phi(z)_{\left[n_{1} n_{2} n_{3}\right]}^{\left[j_{1} j_{2} k\right]} \rightarrow & \left(U_{1} * W_{U_{*}(1)}^{-1}\right)_{k} *\left(U_{2} * W_{U_{*}(2)}^{-1}\right)_{j_{2}^{\prime}}^{j_{2}} *\left(U_{2} * W_{U_{*}(2)}^{-1}\right)_{j_{1}^{\prime}}^{j_{1}} \\
& *\left(W_{U_{*}(2)}\right)_{j_{1}^{\prime \prime}}^{j_{1}^{\prime}} *\left(W_{U_{*}(2)}\right)_{j_{2}^{\prime \prime}}^{j_{2}^{\prime}} *\left(W_{U_{*}(1)}\right)^{k} * \phi(z)_{\left[n_{1}^{\prime \prime} n_{2}^{\prime \prime} n_{3}^{\prime \prime}\right]}^{\left[j_{1 \prime \prime}^{\prime \prime} k\right]} \\
& *\left(W_{U_{*}(3)}^{-1}\right)_{n_{1}^{\prime}}^{n_{1 \prime}^{\prime \prime}} *\left(W_{U_{*}(3)}^{-1}\right)_{n_{2}^{\prime}}^{n_{2}^{\prime \prime}} *\left(W_{U_{*}(3)}^{-1}\right)_{n_{3}^{\prime}}^{n_{\prime \prime}^{\prime \prime}} \\
& *\left(W_{U_{*}(3)} * U_{3}^{-1}\right)_{n_{3}}^{n_{3}^{\prime}} *\left(W_{U_{*}(3)} * U_{3}^{-1}\right)_{n_{2}}^{n_{2}^{\prime}} *\left(W_{U_{*}(3)} * U_{3}^{-1}\right)_{n_{1}}^{n_{1}^{\prime}},
\end{aligned}
$$

where $U_{3}$ is an element of the gauge group $U_{*}(3)$. (The index $k$ is unnecessary, however we chose to use it in order to show that the Higgsac fields carry also $U_{*}(1)$ charge.) Upon the condensation of these superfields the only tr- $U(1)$ field remaining massless is the usual weak hypercharge superfield. 
The Higgsac superfield is also used to give masses to the doublet leptonic superfields $L_{i}^{\prime}$ and $L_{i}^{\prime \prime}$. Indeed, it constitutes the following additional Yukawa couplings in the superpotential with the Wilson lines and the composite Higgsac:

$$
\left(W_{U_{*}(2)} * L^{\prime} * W_{U_{*}(1)}^{-1}\right)^{T} *\left(W_{U_{*}(2)} * L^{\prime \prime}\right) * \Phi_{U_{*}(2) \times U_{*}(1)} .
$$

The condensation of $\Phi_{U_{*}(2) \times U_{*}(1)}$ leads to the spontaneous breaking where the surviving $U(1)^{\prime}$ is a linear combination of $\operatorname{tr}-U(1)$ of $U_{*}(2)$ and $U_{*}(1)$. At the same time the leptonic superfields get a mass of order $\left\langle\Phi_{U_{*}(2) \times U_{*}(1)}\right\rangle$. Thus, these two superfields do not appear at low energies. Of course, similar terms could be written also for any other matter fields in the theory. Note however that the new leptonic fields are vector-like under the SM subgroup of the NC SM gauge group. Thus, decoupling only these fields using the Higgsac field does not lead to anomalies for the standard model gauge group.

\subsection{Charge quantization}

The symmetry reduction mechanism proposed here matches exactly, in the $\Theta \rightarrow 0$ limit, the original Higgsac mechanism proposed in [6]. The gauge-covariant completion given by the Wilson lines insures just that the symmetry breaking is spontaneous and problems with unitarity do not appear [30]. One can check the fractional charge for quarks in the way discussed in [6]. The charge assignments for quarks and the standard model leptons in Table 1 coincide with the ones in the NC SM [16], so they obviously lead to the correct fractional charges under the commutative SM gauge group.

\subsection{Anomaly cancelation}

A solution to the anomaly problem was also given in Ref. [16]. In the $\mathrm{NC} U_{*}(n)$ gauge theory, to cancel the anomaly, the following conditions should hold [9]:

$$
\begin{aligned}
& \operatorname{Tr} T^{a}\left\{T^{b}, T^{c}\right\}=0, \\
& \operatorname{Tr} T^{a}\left[T^{b}, T^{c}\right]=0,
\end{aligned}
$$

where $T^{a}$ is a generator of $U_{*}(n)$ gauge group. The first equation is the condition for anomaly cancelation for the commutative case while the latter is a new condition appearing in the $\mathrm{NC}$ case. In order to satisfy these conditions in the NC SM case, it is sufficient to introduce two leptonic fields $L^{\prime}$ and $L^{\prime \prime}$ whose charge assignments are give in Table 1. For instance, consider the anomaly containing three $U_{*}(2)$ gauge bosons. In this case, above conditions are written by

$$
\begin{aligned}
& \sum_{f} \operatorname{Tr} T^{a}\left\{T^{b}, T^{c}\right\}=d \operatorname{Tr} T^{a}\left\{T^{b}, T^{c}\right\}=0, \\
& \sum_{f} \operatorname{Tr} T^{a}\left[T^{b}, T^{c}\right]=d \operatorname{Tr} T^{a}\left[T^{b}, T^{c}\right]=0,
\end{aligned}
$$


where the sum runs over $U_{*}(2)$ charged matter and it amounts to the constant $d$. Inserting the charge assignments given in Table 1 for $L, Q_{L}, L^{\prime}$ and $L^{\prime \prime}$, we find for each generation

$$
d=1+(-1) \times 3+1+1=0
$$

Similarly, one can check the conditions for anomaly cancelation containing other gauge bosons. Note that we do not have to check the mixed anomaly such as that containing two $U_{*}(3)$ gauge bosons and one $U_{*}(2)$ gauge boson since it does not exist in the NC gauge theory [10]. Thus, one concludes that the matter content of the NC SM differs from ordinary SM by two additional leptonic fields, two Higgsac fields and two additional massive gauge bosons.

The UV/IR mixing which causes problems for the hypercharge $U_{Y}(1)$ gauge field at oneloop level will be considered in detail in Section 3 and a possible solution in a supersymmetric version of the NC SM will be proposed.

Finally we summarize our ( $R$-parity conserving) superpotential:

$$
\begin{aligned}
& \mathcal{W}=\mathcal{W}_{\text {Yukawa }}+\mathcal{W}_{\text {Higgsac }}, \\
& \begin{aligned}
\mathcal{W}_{\text {Yukawa }}= & \lambda_{e}^{i j} H_{1} * L_{i} * E_{j}+\lambda_{u}^{i j} Q_{i} * H_{2} * \bar{U}_{j}+\lambda_{d}^{i j} Q_{i} * H_{3} * \bar{D}_{j} \\
& +\mu_{12} H_{1} * H_{2}+\mu_{34} H_{3} * H_{4}+\lambda_{L^{\prime \prime}}^{i j} H_{1} * L_{i}^{\prime \prime} * E_{j}, \\
\mathcal{W}_{\text {Higgsac }}= & \sum_{a}\left(m^{2} \Phi_{a}-\frac{\lambda}{3} \Phi_{a} * \Phi_{a} * \Phi_{a}\right),
\end{aligned}
\end{aligned}
$$

where the index $a$ denotes the type of the Higgsac superfield defined in (2.32) and (2.34): $a=U_{*}(2) \times U_{*}(1), U_{*}(3) \times U_{*}(2) \times U_{*}(1)$.

\section{Quantum corrections and SUSY breaking}

As we have seen, the model we proposed is supersymmetric, anomaly-free and produces the correct quantized hypercharges for fermions after NC gauge symmetry breaking by super Higgsac fields. To complete the description of the NC MSSM, we need to specify the SUSY breaking. However, as we mentioned in the Introduction, once SUSY is broken, serious problems with the hypercharge $U_{Y}(1)$ gauge field arise, which are caused by the UV/IR mixing. Let us first clarify these problems in detail and then discuss a possible solution.

In order to clarify the problems, in the following we focus on Euclidean SUSY $U_{*}(1)$ gauge theory which was studied in Refs. [12, 19, 14, 31]. Recall that $U_{Y}(1)$ is constructed from the linear combination of $\operatorname{tr}-U(1)$ parts of the $\mathrm{NC}$ gauge groups $U_{*}(n)$. A $U_{*}(1)$ gauge theory involves qualitative features similar to the $\operatorname{tr}-U(1)$ factors, and so involves all essential features of the problems we explain. In this setting at the one loop level the polarization tensor for $U_{*}(1)$ gauge field generally has the form [12, 14, 31]:

$$
\Pi_{m n}=\Pi_{1}\left(k^{2}, \tilde{k}^{2}\right)\left(k^{2} \delta_{m n}-k_{m} k_{n}\right)+\Pi_{2}\left(k^{2}, \tilde{k}^{2}\right) \frac{\tilde{k}_{m} \tilde{k}_{n}}{\tilde{k}^{2}} \quad \text { with } \quad \tilde{k}^{m}=\Theta^{m n} k_{n},
$$




\begin{tabular}{|c|c|c|c|c|}
\hline $\mathrm{j}$ & real scalar & Weyl fermion & gauge boson & ghost \\
\hline$\alpha_{j}$ & $-\frac{1}{2}$ & $\frac{1}{2}$ & $-\frac{1}{2}$ & 1 \\
\hline$C_{j}$ & 0 & $\frac{1}{2}$ & 2 & 0 \\
\hline$d_{j}$ & 1 & 2 & 4 & 1 \\
\hline
\end{tabular}

Table 2: Coefficients in the evaluation of the loop integrals.

where $k^{m}$ is the external momentum. The $\Pi_{1}$ part multiplies the ordinary transverse structure and is related to the gauge coupling by

$$
\frac{1}{g^{2}\left(k^{2}, \tilde{k}^{2}\right)}=\frac{1}{g_{0}^{2}}+\Pi_{1}\left(k^{2}, \tilde{k}^{2}\right) .
$$

The $\Pi_{2}$ part is a new Lorentz symmetry violating structure, which is specific to NC QFT and explicitly depends on $\Theta_{m n}$.

Performing a one loop calculation for the polarization tensor one obtains [14]

$$
\Pi_{m n}(k)=\Pi_{m n}(k, l=0)-\operatorname{Re}\left\{\Pi_{m n}(k, l=\tilde{k})\right\}
$$

with

$$
\begin{gathered}
\Pi_{m n}(k, l)=2 \sum_{j} \alpha_{j} \int \frac{d^{4} q}{(2 \pi)^{4}}\left\{d(j)\left[\frac{(2 p+k)_{m}\left(2_{p}+k\right)_{n}}{\left(p^{2}+m_{j}^{2}\right)\left((p+k)^{2}+m_{j}^{2}\right)}-2 \frac{\delta_{m n}}{p^{2}+m_{j}^{2}}\right]\right. \\
\left.+4 C(j) \frac{k^{2} \delta_{m n}-k_{m} k_{n}}{\left(p^{2}+m_{j}^{2}\right)\left((p+k)^{2}+m_{j}^{2}\right)}\right\} \exp (i p \cdot l),
\end{gathered}
$$

where the coefficients $\alpha_{j}, d(j)$ and $C(j)$ are given in Table 2, and $m_{j}$ are soft SUSY breaking masses. Here the $\Pi_{m n}(k, l=0)$ contribution is the so-called planar part, while $\Pi_{m n}(k, l=\tilde{k})$ is the non-planar part. The exponential factor $\exp (i p \cdot l)$ in the non-planar part gives rise to the UV/IR mixing. This factor follows from the Moyal star products in the Lagrangian. At large value of the internal momentum, the exponential factor removes the divergence of the integral. However, when the external momentum goes to zero, the divergence reappears. Thus the divergence at the UV scales is interpreted as an IR singularity.

In general, both $\Pi_{1}$ and $\Pi_{2}$ are affected by the UV/IR mixing. In the $\Pi_{1}$ part, the effect of the UV/IR mixing is to alter the behavior of running of the $U_{*}(1)$ coupling constant. As $k^{2} \rightarrow 0$, it has the following form

$$
\frac{1}{g\left(k^{2}, \tilde{k}^{2}\right)}=\Pi_{1}\left(k^{2}, \tilde{k}^{2}\right) \rightarrow-\frac{b_{0}}{\left(4 \pi^{2}\right)} \log k^{2},
$$

while for $k^{2} \rightarrow \infty$

$$
\frac{1}{g\left(k^{2}, \tilde{k}^{2}\right)}=\Pi_{1}\left(k^{2}, \tilde{k}^{2}\right) \rightarrow \frac{b_{0}}{\left(4 \pi^{2}\right)} \log k^{2}
$$


where $b_{0}$ is the one-loop beta-function.

The $\Pi_{2}$ part arises purely from noncommutativity, but is known to vanish if the theory has exact SUSY [12]. On the other hand, for nonsupersymmetric gauge theories the $\Pi_{2}$ part is nonzero and causes problems. The most serious one is that it produces an unacceptable infrared singularity $\Pi_{2} \sim 1 / \tilde{k}^{2}$. In theories with soft SUSY breaking terms, thanks to the equal number of bosonic and fermionic degrees of freedom, this infrared singularity is cancelled [12]. However, a subleading finite term still remains :

$$
\Pi_{2} \sim \Delta M_{S U S Y}^{2}, \quad \Delta M_{S U S Y}^{2}=\frac{1}{2} \sum_{s} M_{s}^{2}-\sum_{f} M_{f}^{2}
$$

unless SUSY is exact. This produces vacuum birefringence, i.e. a mass to only one of the polarizations of the $U_{*}(1)$ gauge field, leaving the other polarization massless. Furthermore, a negative $\Pi_{2}$ would lead to tachyons while a positive mass is phenomenologically strongly constrained. Consequently, it would seem that the $U_{Y}(1)$ gauge field, which is a linear combination of $\operatorname{tr}-U_{*}(1)$ fields, has serious problems already at one-loop level [13, 15].

One way to avoid these problems is to modify the UV physics [32]. In the above analysis, it is assumed that the NC QFT is valid up to arbitrarily large momentum scales. However, if $\mathrm{NC}$ QFT is realized as a low energy effective theory of some underlying theory such as string theory, the theory should be modified above some UV scale, e.g. string scale. Indeed, since NC QFT is realized as a special limit of open strings in a background of antisymmetric tensor field $B_{m n}$, it is expected that at least above the string scale the $B_{m n}$ does not have a vacuum expectation value and the noncommutativity does not appear there. This modification affects the low energy physics since the infrared region receives the effect from the UV domain due to the UV/IR mixing. The modification actually improves the situation, leading to the birefringence effect. In Ref. [32, it is shown that constraints on this effect require the noncommutativity scale to be close to the Planck scale. Furthermore, in this setting the behavior of the running coupling constant is exactly the same with the commutative one at the low energies smaller than some infrared scale specified by $\Lambda_{\mathrm{IR}} \sim \Lambda_{\mathrm{UV}}^{2} / M_{\mathrm{NC}}$. This is a desired property for considering phenomenological model building although the photon may be still tachyonic (the tachyonic mode is possible since the Lorentz symmetry is now broken).

Here we briefly discuss another possibility for improving the above situation. We assume that SUSY in the theory is spontaneously broken by a mechanism such as O'Raifeartaigh and Fayet-Iliopoulos mechanisms. The breaking of SUSY is assumed to occur at a hidden sector, so that its effect on the visible MSSM sector is the occurrence of soft SUSY breaking masses $m_{i}$ below some SUSY breaking scale $M_{\text {SUSY }}$. Above this scale, SUSY is expected to be restored and all the soft SUSY breaking terms to vanish. Recalling that the $\Pi_{2}$ part vanishes in theories with exact SUSY, it is expected that the above situation renders the $\Pi_{2}$ contribution small so that the one-loop correction to the polarization tensor is consistent with the experimental bound for the Lorentz violation. To be more precise we also need to consider the relation between $M_{\mathrm{SuSY}}$ and the NC scale $M_{\mathrm{NC}} \sim|\Theta|^{-1 / 2}$.

In order to estimate the one-loop contribution to $\Pi_{2}$ in the setting mentioned above, we 
divide the integral (3.4) into two parts

$$
\begin{aligned}
\Pi_{m n} & =\left(\Pi_{a}\right)_{m n}+\left(\Pi_{b}\right)_{m n} \\
& =\left(\int_{0 \leq|p| \leq M_{\mathrm{SUSY}}}\left(m_{j} \neq 0\right)+\int_{M_{\mathrm{SUSY}} \leq|p| \leq \infty}\left(m_{j}=0\right)\right) \frac{d^{4} p}{(2 \pi)^{4}} \cdots,
\end{aligned}
$$

where the ellipsis denotes the integrand. Here we treat the soft SUSY breaking mass term as a step function like, i.e. $m_{j}=0\left(M_{\mathrm{SUSY}} \leq|p| \leq \infty\right)$ and $m_{j} \neq 0\left(0 \leq|p| \leq M_{\mathrm{SUSY}}\right)$. Note that in this treatment gauge invariance gets lost since $M_{\mathrm{SUSY}}$ behaves as a cut-off which leads to a non-gauge invariant term in the calculation. However, we simply ignore the non-gauge invariant term in what follows. Our purpose is to see whether this setting can solve the problem of $\operatorname{tr}-U(1)$ part. We believe that this qualitative argument does not change in a proper gauge invariant regularization and we postpone a more rigorous analysis to a future work. The first integral $\left(\Pi_{a}\right)_{m n}$ in (3.8) can be written as [14]:

$$
\begin{aligned}
\left(\Pi_{a}\right)_{m n}(k)= & \frac{1}{4 \pi^{2}}\left(k^{2} \delta_{m n}-k_{m} k_{n}\right) \\
& \times \sum_{j} \alpha_{j} \int_{0}^{1} d x[4 C(j)-(1-2 x) d(j)]\left[K_{0}\left(\frac{\sqrt{A_{j}}}{M_{\mathrm{SUSY}}}\right)-K_{0}\left(\frac{\sqrt{A_{j}}}{M_{\mathrm{eff}}}\right)\right] \\
+ & \frac{1}{4 \pi^{2}} \tilde{k}_{m} \tilde{k}_{n} M_{\mathrm{eff}}^{2} \sum_{j} \alpha_{j} d(j) \int_{0}^{1} d x A_{j} K_{2}\left(\frac{\sqrt{A_{j}}}{M_{\mathrm{eff}}}\right) \\
+ & \delta_{m n}[\text { gauge non-invariant term }],
\end{aligned}
$$

where

$$
A_{j}=m_{j}^{2}+x(1-x) k^{2}, \quad \frac{1}{M_{\mathrm{eff}}^{2}}=\frac{1}{M_{\mathrm{SUSY}}^{2}}+\tilde{k}^{2} .
$$

The non-planar part in the second integral $\left(\Pi_{b}\right)_{m n}$ in $(3.8)$ is exactly zero because SUSY is manifest there and therefore only the first integral will contribute to the birefringence effect. Using (3.9) one can obtain the following approximate expressions for $\Pi_{2}$ part from (3.8) [32]

$$
\Pi_{2}=\left\{\begin{array}{l}
D \Delta M_{\mathrm{SUSY}}^{2}, \quad \text { for } \frac{M_{\mathrm{NC}}^{2}}{M_{\mathrm{SUSY}}} \ll k \ll \Delta M_{\mathrm{SUSY}}, \\
D^{\prime} \Delta M_{\mathrm{SUSY}}^{2} M_{\mathrm{SUSY}}^{2} \tilde{k}^{2}, \quad \text { for } k \ll \frac{M_{\mathrm{NC}}^{2}}{M_{\mathrm{SUSY}}}, \quad m_{j}^{2} \ll M_{\mathrm{SUSY}}^{2},
\end{array}\right.
$$

where $D$ and $D^{\prime}$ are known constants. One can see that the second equation in (3.11) is suppressed by the NC scale $M_{\mathrm{NC}}$ while the first equation does not have any suppression. In the following we consider only the second expression, which indeed gives a promising result.

Following the discussion in Ref. [15], we consider the equation of motion for the photon,

$$
\Pi^{m n}(k) A_{n}(k)=0 .
$$


We specify the noncommutativity as $\Theta^{13}=-\Theta^{31}=\Theta=1 / M_{\mathrm{NC}}^{2}$, while all other components of $\Theta^{m n}$ are taken to be zero. The photon propagates in the third direction $k^{\mu}=\left(k^{0}, 0,0, k^{3}\right)$. Now we have two polarization vectors

$$
A_{1}^{m}=(0,1,0,0), \quad A_{2}^{m}=(0,0,1,0) .
$$

Substituting this into Eq. (3.12), we find

$$
\begin{aligned}
\left(\Pi_{1} k^{2}-\Pi_{2}\right) A_{1}^{m} & =0, \\
\Pi_{1} k^{2} A_{2}^{m} & =0 .
\end{aligned}
$$

The second equation representing the equation of motion for a polarized photon along $A_{2}^{m}$ behaves like ordinary photon while the first equation for polarized photon $A_{1}^{m}$ receives a new effect, $\Pi_{2}$. Now in order to study in more detail we substitute the expression (3.11) into (3.14). Then we obtain

$$
k^{2}+D^{\prime} \frac{\Delta M_{\mathrm{SUSY}}^{2} M_{\mathrm{SUSY}}^{2}}{\Pi_{1} M_{\mathrm{NC}}^{4}}\left(k^{3}\right)^{2}=0, \quad \text { for } k \ll \frac{M_{\mathrm{NC}}^{2}}{M_{\mathrm{SUSY}}}, \quad m_{j}^{2} \ll M_{\mathrm{SUSY}}^{2} .
$$

Now we consider the dispersion relation of photon for the case (3.16). Restoring the light speed $c$ and using $k^{0}=\omega$ for the frequency of the wave, we find

$$
\omega^{2}-c^{2}\left(\frac{1}{1+\Delta n}\right)^{2}\left(k^{3}\right)^{2}=0
$$

with

$$
\Delta n \simeq \frac{D^{\prime}}{2 \Pi_{1}} \frac{\Delta M_{\mathrm{SUSY}}^{2} M_{\mathrm{SUSY}}^{2}}{M_{\mathrm{NC}}^{4}}
$$

where $D^{\prime}=1 / 4 \pi^{2}$. In Ref. [33], all possible dimension four Lorentz violating operators in electrodynamics were studied and the constraints were obtained. The Lorentz violating operators can be related to our $\Delta n$ [32]. Most strongest bound in Ref. [33] is obtained from observation of objects at cosmological distances

$$
\left|\Delta n_{\text {cosmo }}\right| \leq 10^{-37}-10^{-32} .
$$

Our result is consistent with this bound, for instance, if we take the following values for scales: $M_{\mathrm{SUSY}} \sim 10^{10}, M_{\mathrm{NC}} \sim 10^{18}, m_{j} \sim 10^{2}$ and $k \sim 100 \mathrm{GeV}$. In the above setting, the $\Delta n$ is found to be

$$
\Delta n \sim 10^{-62} .
$$

Our setting is consistent with the experimental bound for the Lorentz violation although the photon may be tachyonic similarly to the case in Ref. [32]. 
We briefly summarize our argumentation. At scales above $M_{\mathrm{SUSY}}$, SUSY is exact and thus the contribution to $\Pi_{2}$ from these scales is exactly cancelled. Furthermore, if $M_{\mathrm{SusY}}$ is much smaller than the scale of noncommutativity, the contribution from scales below $M_{\mathrm{SUSY}}$ is expected to be negligible because in this region the theory should be effectively commutative.

Note that in our case the behaviour of $U(1)$ running coupling constant is still altered by the $\mathrm{UV} / \mathrm{IR}$ mixing compared to the commutative one, while in the theory with the UV completion in [32 the running behaviour is the same with the commutative one below the scale $\Lambda_{\text {IR }} \sim$ $\Lambda_{\mathrm{UV}}^{2} / M_{\mathrm{NC}}$. We leave a further study on the behaviour of the running coupling constant for later work.

\section{Summary and discussion}

In this paper, we have proposed a NC version of MSSM. The guiding principles in constructing the action are to require SUSY, NC gauge invariance, absence of anomalies and correct fractional $U_{Y}(1)$ charges for fermions. The NC gauge invariance requires to introduce two extra gauge fields and their superpartners compared to the MSSM gauge field content, since our model is based on the $\mathrm{NC}$ gauge groups $U_{*}(3) \times U_{*}(2) \times U_{*}(1)$. Other requirements lead us to introducing two new Higgs superfields $H_{3}, H_{4}$ for the construction of the Yukawa couplings and two leptonic superfields $L^{\prime}, L^{\prime \prime}$ for the cancellation of the anomaly, compared to the commutative MSSM matter content.

Further additional matter, the Higgsac superfield, is introduced. This plays two roles in our model. One of them is to reduce the $\mathrm{NC}$ gauge symmetry from $U_{*}(3) \times U_{*}(2) \times U_{*}(1)$ to the $\mathrm{SM}$ one. The two extra gauge bosons and their superpartners are decoupled at low energies via the condensation of the Higgsac superfield, which gives masses to the extra gauge fields. The other role is to achieve the decoupling of the extra leptonic superfields. The Higgsac superfield can form Yukawa coupling with these two leptonic superfields through the NC Wilson lines. Upon the condensation of the Higgsac, the leptons become massive and thus they decouple at low energies.

We have discussed the quantum properties of our model, especially concerning the hypercharge $U_{Y}(1)$ sector. In the NC SM proposed in Ref. 6], the hypercharge $U_{Y}(1)$ gauge field suffers from the problems on the tachyonic mass and the vacuum birefringence once the oneloop corrections are taken into account. The $U_{Y}(1)$ gauge group is a linear combination of three $\operatorname{tr}-U(1)$ gauge groups which are subgroups of the $U_{*}(n)$ gauge groups. In the NC setting, the $\operatorname{tr}-U(1)$ gauge field is affected by the UV/IR mixing and it generates a Lorentz violating term in the polarization tensor. As a result, $\operatorname{tr}-U(1)$ gauge field has the serious problems mentioned above. In this paper, we gave a possible solution to this problem, by assuming that supersymmetry is restored above the SUSY breaking scale $M_{\mathrm{SUSY}}$. Then, with appropriate values of the scales $M_{\mathrm{SUSY}}, M_{\mathrm{NC}}$ and $m_{j}$, the Lorentz violating term in the polarization tensor has small enough value to avoid violating experimental bounds.

Note that our model does not go to the commutative MSSM in the limit of vanishing 
noncommutativity parameter $\Theta \rightarrow 0$ at the tree level since we introduced two new Higgs superfields $H_{3}$ and $H_{4}$ and the interactions with these fields do not vanish in this limit (other terms including the new leptonic fields do not appear at low energies as mentioned above). This implies that our model involves many interesting new phenomenological features compared to the commutative MSSM.

One of the most important things to study in the low energy physics of our model is the electroweak symmetry breaking. In our model, since a new down-type Higgs $H_{3}$ is introduced beside $H_{4}$, these fields must obtain vacuum expectation values in order to give masses to downtype quarks while $H_{1}$ and $H_{2}$ corresponding to the usual Higgses appearing in the commutative MSSM have to condensate in order to give masses to up-type quarks and leptons. In the commutative MSSM with soft SUSY breaking terms, electroweak symmetry breaking does not occur at the tree level, but taking one-loop corrections to Higgs mass into account, the Higgs mass runs in the infrared and eventually goes to negative 34. As a result, the Higgs field has a nonzero vacuum expectation value and electroweak symmetry breaking occurs. This mechanism may be also applied in our model. We postpone a more detailed study of this issue for future work. It would also be interesting to extend NC version of the SM [23] using Seiberg-Witten map to the supersymmetric case.

\section{Acknowledgements}

We are grateful to Masud Chaichian for illuminating discussions and careful reading of the manuscript. We thank Adi Armoni, Valentin V. Khoze, Archil Kobakhidze and M. M. SheikhJabbari for useful correspondence. The work of M.A. is supported by the bilateral program of Japan Society for the Promotion of Science and Academy of Finland, "Scientist Exchanges".

S.S. acknowledges a grant from GRASPANP, the Finnish Graduate School in Particle and Nuclear Physics.

\section{References}

[1] N. Seiberg and E. Witten, JHEP 9909 (1999) 032 arXiv:hep-th/9908142.

[2] R. J. Szabo, Phys. Rept. 378 (2003) 207 arXiv:hep-th/0109162.

[3] M. R. Douglas and N. A. Nekrasov, Rev. Mod. Phys. 73 (2001) 977 arXiv:hep-th/0106048.

[4] S. Terashima, Phys. Lett. B 482 (2000) 276 arXiv:hep-th/0002119.

[5] M. Chaichian, P. Prešnajder, M. M. Sheikh-Jabbari and A. Tureanu, Phys. Lett. B 526 (2002) 132 arXiv:hep-th/0107037. 
[6] M. Chaichian, P. Prešnajder, M. M. Sheikh-Jabbari and A. Tureanu, Eur. Phys. J. C 29 (2003) 413 arXiv:hep-th/0107055.

[7] M. Hayakawa, Phys. Lett. B 478 (2000) 394 [arXiv:hep-th/9912094]; "Perturbative analysis on infrared and ultraviolet aspects of noncommutative QED on $\mathrm{R}^{4}$," arXiv:hep-th/9912167.

[8] J. L. Hewett, F. J. Petriello and T. G. Rizzo, Phys. Rev. D 66 (2002) 036001 arXiv:hep-ph/0112003.

[9] J. M. Gracia-Bondia and C. P. Martin, Phys. Lett. B 479 (2000) 321 arXiv:hep-th/0002171]; L. Bonora, M. Schnabl and A. Tomasiello, Phys. Lett. B 485 (2000) 311 |arXiv:hep-th/0002210]; F. Ardalan and N. Sadooghi, Int. J. Mod. Phys. A 16 (2001) 3151 [arXiv:hep-th/0002143]; Int. J. Mod. Phys. A 17 (2002) 123 arXiv:hep-th/0009233; A. Armoni, E. Lopez and S. Theisen, JHEP 0206 (2002) 050 arXiv:hep-th/0203165.

[10] C. P. Martin, Nucl. Phys. B 623 (2002) 150 arXiv:hep-th/0110046.

[11] V. V. Khoze and J. Levell, JHEP 0409 (2004) 019 [arXiv:hep-th/0406178].

[12] A. Matusis, L. Susskind and N. Toumbas, JHEP 0012 (2000) 002 [arXiv:hep-th/0002075.

[13] A. Armoni and E. Lopez, Nucl. Phys. B 632 (2002) 240 arXiv:hep-th/0110113.

[14] L. Alvarez-Gaume and M. A. Vazquez-Mozo, Nucl. Phys. B 668 (2003) 293 arXiv:hep-th/0305093].

[15] J. Jaeckel, V. V. Khoze and A. Ringwald, JHEP 0602 (2006) 028 arXiv:hep-ph/0508075.

[16] M. Chaichian, A. Kobakhidze and A. Tureanu, Eur. Phys. J. C 47 (2006) 241 arXiv:hep-th/0408065.

[17] A. Armoni, Nucl. Phys. B 593 (2001) 229 arXiv:hep-th/0005208.

[18] L. Bonora and M. Salizzoni, Phys. Lett. B 504 (2001) 80 arXiv:hep-th/0011088.

[19] C. E. Carlson, C. D. Carone and R. F. Lebed, Phys. Lett. B 549 (2002) 337 arXiv:hep-ph/0209077.

[20] H. O. Girotti, M. Gomes, V. O. Rivelles and A. J. da Silva, Nucl. Phys. B 587 (2000) 299. arXiv:hep-th/0005272.

[21] M. Chaichian, P. P. Kulish, K. Nishijima and A. Tureanu, Phys. Lett. B 604 (2004) 98 arXiv:hep-th/0408069|; M. Chaichian, P. Prešnajder and A. Tureanu, Phys. Rev. Lett. 94 (2005) 151602 arXiv:hep-th/0409096.

[22] Y. Kobayashi and S. Sasaki, Int. J. Mod. Phys. A 20 (2005) 7175 arXiv:hep-th/0410164; M. Ihl and C. Saemann, JHEP 0601 (2006) 065 [arXiv:hep-th/0506057]. 
[23] X. Calmet, B. Jurčo, P. Schupp, J. Wess and M. Wohlgenannt, Eur. Phys. J. C 23 (2002) 363 [arXiv:hep-ph/0111115].

[24] J. Wess and J. Bagger, "Supersymmetry and supergravity," Princeton Univ. Press, Princeton (1992).

[25] T. W. B. Kibble, Phys. Rev. 155 (1967) 1554.

[26] D. J. Gross, A. Hashimoto and N. Itzhaki, Adv. Theor. Math. Phys. 4 (2000) 893 arXiv:hep-th/0008075.

[27] C. S. Chu and H. Dorn, Phys. Lett. B 524 (2002) 389 arXiv:hep-th/0110147]; C. S. Chu, V. V. Khoze and G. Travaglini, Phys. Lett. B 543 (2002) 318 [arXiv:hep-th/0112139].

[28] S. Marculescu and L. Mezincescu, Nucl. Phys. B 181 (1981) 127; R. L. Mkrtchian, Nucl. Phys. B 198, 295 (1982); M. Awada and F. Mansouri, Phys. Lett. B 384 (1996) 111 arXiv:hep-th/9512098.

[29] F. Ardalan and N. Sadooghi, Int. J. Mod. Phys. A 20 (2005) 2859 arXiv:hep-th/0307155.

[30] Work in progress.

[31] V. V. Khoze and G. Travaglini, JHEP 0101 (2001) 026 [arXiv:hep-th/0011218].

[32] S. A. Abel, J. Jaeckel, V. V. Khoze and A. Ringwald, JHEP 0609 (2006) 074 arXiv:hep-ph/0607188].

[33] V. A. Kostelecky and M. Mewes, Phys. Rev. D 66 (2002) 056005 arXiv:hep-ph/0205211.

[34] K. Inoue, A. Kakuto, H. Komatsu and S. Takeshita, Prog. Theor. Phys. 67 (1982) 1889. 